Homology, Homotopy and Applications, vol.13(2), 2011, pp.1-17

\title{
LIFTING OF MODEL STRUCTURES TO FIBRED CATEGORIES
}

\author{
ABHISHEK BANERJEE \\ (communicated by Charles A. Weibel)
}

\begin{abstract}
A fibred category consists of a functor $p: \mathbf{N} \longrightarrow \mathbf{M}$ between categories $\mathbf{N}$ and $\mathbf{M}$ such that objects of $\mathbf{N}$ may be "pulled back along any arrow of $\mathbf{M}$ ". Given a fibred category $p: \mathbf{N} \longrightarrow \mathbf{M}$ and a model structure on the "base category" $\mathbf{M}$, we show that there exists a lifting of the model structure on $\mathbf{M}$ to a model structure on $\mathbf{N}$. We will refer to such a system as a "fibred model category" and give several examples of such structures. We show that, under certain conditions, right homotopies of maps in the base category $\mathbf{M}$ may be lifted to right homotopic maps in the fibred category. Further, we show that these lifted model structures are well behaved with respect to Quillen adjunctions and Quillen equivalences. Finally, we show that if $\mathbf{N}$ and $\mathbf{M}$ carry compatible closed monoidal structures and the functor $p$ commutes with colimits, then a Quillen pair on $\mathbf{M}$ lifts to a Quillen pair on $\mathbf{N}$.
\end{abstract}

\section{Introduction}

Model structures, first introduced by Quillen [1], provide an axiomatic setup for Homotopy Theory. The category of chain complexes of modules over a ring, the category of simplicial sets, the category of modules over a Frobenius ring and the category of topological spaces all carry well-known model category structures (see, for instance, [2]). The pursuit of defining model structures has been carried on to various other categories, such as the category of modules over a group ring $K[G]$ ( $K$ being a commutative Gorenstein ring and $G$ a finite group) (see Hovey [3]), the category of chain complexes of quasi-coherent sheaves over a scheme (see Gillespie [4]) and the category of simplicial presheaves (see Jardine [5]).

In this paper, we introduce a method for defining model structures on a large class of categories by describing a process whereby a model structure may be lifted from a "base category" $\mathbf{M}$ to a category $\mathbf{N}$ "fibred over" $\mathbf{M}$ via a functor $p: \mathbf{N} \longrightarrow \mathbf{M}$. Fibred categories, which first appeared in SGA-I [6], provide just the right axiomatic framework for studying categories such as the category of presheaves over a given category or the category of vector bundles over topological spaces.

Received May 8, 2011; published on September 9, 2011.

2000 Mathematics Subject Classification: 55U35.

Key words and phrases: fibred category, model category.

Article available at http://intlpress.com/HHA/v13/n2/a1 and doi:10.4310/HHA.2011.v13.n2.a1

Copyright (C) 2011, International Press. Permission to copy for private use granted. 
Consider, for instance, the categories $\mathbf{V B}_{n}, n \geqslant 1$ of $n$-dimensional (say complex) vector bundles over the category Top of topological spaces. A map $f: X \longrightarrow Y$ of such spaces allows us to choose a pullback functor (unique up to isomorphism) $f^{*}: \mathbf{V B}_{n}(Y) \longrightarrow \mathbf{V B}_{n}(X)$ from the category $\mathbf{V B}_{n}(Y)$ of $n$-dimensional vector bundles over $Y$ to the category $\mathbf{V B}_{n}(X)$ of $n$-dimensional vector bundles over $X$. Hence, the category $\mathbf{V B}_{n}$ is naturally "fibred over" (in a sense we will make precise in Definition 2.2) the category of topological spaces. The choice that is made in the construction of the pullback functor $f^{*}$ is referred to as "choosing a clivage" (see Definition 2.4) for the fibred category $\mathbf{V B}_{n}$ over Top. Besides, the category Top of topological spaces carries a model structure. This situation suggests to us the notion of a "fibred model category" $p: \mathbf{N} \longrightarrow \mathbf{M}$, where $\mathbf{N}$ is a fibred category over $\mathbf{M}$ in the sense of SGA-I $[\mathbf{6}]$ and the "base category" $\mathbf{M}$ is a model category. This notion is introduced in Definition 3.1. Thereafter, in Section 3, using a series of lemmas, we show how we can lift the model structure from the category $\mathbf{M}$ to construct a model structure on the category $\mathbf{N}$. We also show that right homotopic maps in the base category $\mathbf{M}$ can be lifted to right homotopic maps in the lifted model structure on $\mathbf{N}$.

Several examples of lifted model structures are given in Section 3. For instance, let $\mathbf{M}$ be a model category, $U$ an object of $\mathbf{M}$ and $\mathbf{M} / U$ the category of "objects of $\mathbf{M}$ over $U$ ". Then, we show that $\mathbf{M} / U$ carries a natural model structure, which is lifted from $\mathbf{M}$ in the sense mentioned above. This construction generalizes to 2-categories: given a 2-category whose underlying 1-category is a model category, we exhibit a model structure on $\mathbf{M} / V$ for each object $V$ of $\mathbf{M}$.

Finally, in Section 4, we show that the lifted model structures are well behaved with respect to Quillen adjunctions and Quillen equivalences on the "base category" $\mathbf{M}$. We then consider the situation in which $\mathbf{N}$ and $\mathbf{M}$ carry closed monoidal structures compatible with the functor $p: \mathbf{N} \longrightarrow \mathbf{M}$. In this case we show that if the monoidal structure on $\mathbf{M}$ defines a Quillen pair (see $[\mathbf{2}, \S 4.2 .1]$ ) for the model structure on $\mathbf{M}$, then the closed monoidal structure on $\mathbf{N}$ also defines a Quillen pair for the lifted model structure, provided the functor $p$ commutes with colimits.

Our notion of fibred category will be that of [7], which is equivalent to that of SGA-I [6]. The definition of fibred categories is briefly recalled in Section 2 for the convenience of the reader. Throughout this paper, we will sometimes abuse notation by writing $U \in \mathbf{C}$ when we mean that $U$ is an object of the category $\mathbf{C}$.

\section{Fibred categories}

We will now briefly recall the definition of fibred categories and related notions. Our exposition will basically follow that of Canonaco [7]. We start by defining the notion of a cartesian morphism (see $[\mathbf{7}, \S 3.1 .1]$ or $[\mathbf{8}$, Definition 3.1]).

Definition 2.1. Let $p: \mathbf{N} \longrightarrow \mathbf{M}$ be a functor between categories $\mathbf{N}$ and $\mathbf{M}$. Let $\phi: \xi \longrightarrow \eta$ be a morphism in $\mathbf{N}$ and suppose that $p(\xi)=U \in \mathbf{M}, p(\eta)=V \in \mathbf{M}$ and that $p(\phi)=f: U \longrightarrow V$, a morphism in $\mathbf{M}$. Then, we say that the morphism $\phi: \xi \longrightarrow \eta$ is cartesian over $f: U \longrightarrow V$ if, given any morphism $\phi^{\prime}: \xi^{\prime} \longrightarrow \eta$ of $\mathbf{N}$ such that there exists $g: U^{\prime}=p\left(\xi^{\prime}\right) \longrightarrow U$ with $f \circ g=p\left(\phi^{\prime}\right)=f^{\prime}: U^{\prime}=p\left(\xi^{\prime}\right) \longrightarrow$ 
$V=p(\eta)$, there exists a unique morphism $\psi: \xi^{\prime} \longrightarrow \xi$ in $\mathbf{N}$ such that $\phi^{\prime}=\phi \circ \psi$ and $p(\psi)=g$.

Given a functor $p: \mathbf{N} \longrightarrow \mathbf{M}$ and any object $U$ of $\mathbf{M}$, we will denote by $\mathbf{N}_{U}$ the subcategory of $\mathbf{N}$ whose objects are all objects $\eta$ of $\mathbf{N}$ such that $p(\eta)=U$ and whose morphisms are all morphisms $\phi$ of $\mathbf{N}$ such that $p(\phi)=i d_{U}$. Thereafter, we define (see, for instance, $[\mathbf{7}, \S 3.1 .8])$ :

Definition 2.2. The functor $p: \mathbf{N} \longrightarrow \mathbf{M}$ is said to be a fibred category over $\mathbf{M}$ if, given any morphism $f: U \longrightarrow V$ in $\mathbf{M}$, for every $\eta$ in $\mathbf{N}_{V}$, there exists a morphism $\phi: \xi \longrightarrow \eta$ in $\mathbf{N}$ such that $p(\phi)=f$ and $\phi$ is cartesian over $f$.

We also prove here the following statement that will be useful to us in Section 3.

Lemma 2.3. Let $p: \mathbf{N} \longrightarrow \mathbf{M}$ be a fibred category. Suppose that the morphism $\phi^{\prime}:$ $\xi^{\prime} \longrightarrow \eta^{\prime}$ is a retract of the morphism $\phi: \xi \longrightarrow \eta$ in $\mathbf{N}$. Then, if $\phi$ is cartesian over the morphism $p(\phi)$ in $\mathbf{M}, \phi^{\prime}$ is cartesian over the morphism $p\left(\phi^{\prime}\right)$ in $\mathbf{M}$.

Proof. Since $\phi^{\prime}$ is a retract of $\phi$, we must have a commutative diagram (1) in N:

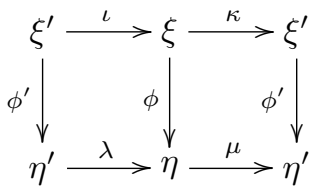

in which the horizontal compositions are identity maps. We want to show that $\phi^{\prime}$ is cartesian over $p\left(\phi^{\prime}\right)$. Suppose, therefore, that we have a morphism $\nu: \chi \longrightarrow \eta^{\prime}$ such that $p(\nu): p(\chi) \longrightarrow p\left(\eta^{\prime}\right)$ factors through $p\left(\phi^{\prime}\right)$, i.e., we have a morphism $s: p(\chi) \longrightarrow$ $p\left(\xi^{\prime}\right)$ such that $p(\nu)=p\left(\phi^{\prime}\right) \circ s$.

Consider the morphism $\lambda \circ \nu: \chi \longrightarrow \eta$. Then $p(\lambda \circ \nu)=p(\lambda) \circ p(\nu)=p(\lambda) \circ p\left(\phi^{\prime}\right)$ $\circ s=p(\phi) \circ p(\iota) \circ s$ factors through $p(\phi)$. Since $\phi$ is cartesian over $p(\phi)$, it follows that there exists a unique $\theta: \chi \longrightarrow \xi$ such that $\lambda \circ \nu=\phi \circ \theta$ and $p(\theta)=p(\iota) \circ s$.

We consider the morphism $\kappa \circ \theta: \chi \longrightarrow \xi^{\prime}$. We note that

$$
\phi^{\prime} \circ(\kappa \circ \theta)=\mu \circ \phi \circ \theta=\mu \circ \lambda \circ \nu=\nu
$$

and

$$
p(\kappa \circ \theta)=p(\kappa) \circ p(\theta)=p(\kappa) \circ p(\iota) \circ s=s .
$$

From (2) and (3) it follows that $\nu: \chi \longrightarrow \eta^{\prime}$ factors through $\phi^{\prime}: \xi^{\prime} \longrightarrow \eta^{\prime}$ as $\nu=$ $\phi^{\prime} \circ(\kappa \circ \theta)$ and that $p(\kappa \circ \theta)=s$.

Hence, in order to show that $\phi^{\prime}$ is cartesian over $p\left(\phi^{\prime}\right)$, it remains to show that the factoring $\nu=\phi^{\prime} \circ(\kappa \circ \theta)$ is unique. Therefore, suppose that $\nu=\phi^{\prime} \circ \tau$ for some morphism $\tau$ in $\mathbf{N}$ such that $p(\tau)=s$. Then $\phi \circ \theta=\lambda \circ \nu=\lambda \circ \phi^{\prime} \circ \tau=\phi \circ \iota \circ \tau$. Further, $p(\iota \circ \tau)=p(\iota) \circ p(\tau)=p(\iota) \circ s=p(\theta)$. But, since the $\phi$ is cartesian over $p(\phi)$, it follows from the uniqueness condition in Definition 2.1 that $\iota \circ \tau=\theta$. Then, $\tau=$ $(\kappa \circ \iota) \circ \tau=\kappa \circ \theta$.

Definition 2.4. A clivage $c$ for a fibred category $p: \mathbf{N} \longrightarrow \mathbf{M}$ is obtained by choosing, for each morphism $f: U \longrightarrow V$ of $\mathbf{M}$ and each object $\eta$ of $\mathbf{N}_{V}$, a morphism $c_{f}(\eta): f^{*}(\eta) \longrightarrow \eta$ of $\mathbf{N}$ that is cartesian over $f$. The clivage is said to be normalised 
if the cartesian morphism chosen over any identity map in $\mathbf{M}$ is also an identity map in $\mathbf{N}$, i.e., $c_{1_{p(\eta)}}(\eta)=1_{\eta}: \eta \longrightarrow \eta$ for any object $\eta$ of $\mathbf{N}$.

Henceforth, unless otherwise mentioned, we will always assume that any clivage we use is normalised. Given a clivage $c$, note that it follows from the definitions that if $\psi: \eta^{\prime} \longrightarrow \eta$ is a morphism in $\mathbf{N}_{V}$, then there exists a corresponding morphism $f^{*}(\psi): f^{*}\left(\eta^{\prime}\right) \longrightarrow f^{*}(\eta)$ in $\mathbf{N}_{U}$ such that $c_{f}(\eta) \circ f^{*}(\psi)=\psi \circ c_{f}\left(\eta^{\prime}\right)$. It follows that, given a fixed clivage $c$, a morphism $f: U \longrightarrow V$ induces a functor

$$
f^{*}: \mathbf{N}_{V} \longrightarrow \mathbf{N}_{U} \text {. }
$$

More generally, given a commutative square in $\mathbf{M}$ as in Figure 1 of (4) and a morphism $\psi: \eta^{\prime} \longrightarrow \eta$ in $\mathbf{N}$ with $p(\psi)=g: V^{\prime} \longrightarrow V:$

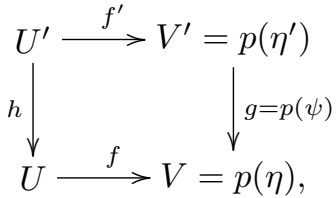

Figure 1.

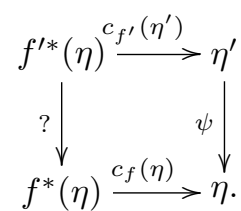

Figure 2.

Since $p\left(\psi \circ c_{f^{\prime}}\left(\eta^{\prime}\right)\right)=g \circ f^{\prime}=f \circ h$ and $c_{f}(\eta): f^{*}(\eta) \longrightarrow \eta$ is cartesian over $f$, there exists a unique morphism $\phi: f^{\prime *}\left(\eta^{\prime}\right) \longrightarrow f^{*}(\eta)$ in $\mathbf{N}$ such that $\psi \circ c_{f^{\prime}}\left(\eta^{\prime}\right)=c_{f}(\eta)$ $\circ \phi$ and $p(\phi)=h$. In other words, given a fixed clivage $c$, the cartesian morphism $c_{f}(\eta): f^{*}(\eta) \longrightarrow \eta$, chosen over any morphism $f: U \longrightarrow V$ in $\mathbf{M}$ with $\eta \in \mathbf{N}$ such that $p(\eta)=V$, is functorial on the category of morphisms of $\mathbf{M}$. We will use this fact later in the proof of Lemma 3.3.

The following alternate description will come in very handy: a pair $\underline{\mathbf{N}}=(p: \mathbf{N} \longrightarrow$ $\mathbf{M}, c)$, consisting of a fibred category $p: \mathbf{N} \longrightarrow \mathbf{M}$ and a chosen normalised clivage $c$, consists of the following data:

(a) For each object $U$ of $\mathbf{M}$, a category $\mathbf{N}(U):=\mathbf{N}_{U}$;

(b) For each morphism $f: U \longrightarrow V$, a functor $\mathbf{N}(f):=f^{*}: \mathbf{N}(V) \longrightarrow \mathbf{N}(U)$ and;

(c) For each pair $f: U \longrightarrow V, g$ : $U^{\prime} \longrightarrow U$ of composable morphisms, natural isomorphisms $c_{g, f}: \mathbf{N}(g) \circ \mathbf{N}(f) \longrightarrow \mathbf{N}(f \circ g)$ of functors.

The isomorphisms in (c) are natural in the following sense: Let $R$ : $\mathbf{C}^{\prime} \longrightarrow \mathbf{C}$, $F, G: \mathbf{C} \longrightarrow \mathbf{D}, S: \mathbf{D} \longrightarrow \mathbf{D}^{\prime}$ be functors and let $c: F \longrightarrow G$ be a natural transformation of functors. Then $c$ induces a natural transformation of functors from $F \circ R$ to $G \circ R$ (resp. from $S \circ F$ to $S \circ G$ ), which we denote by $c * 1_{R}: F \circ R \longrightarrow G \circ R$ (resp. by $1_{S} * c: S \circ F \longrightarrow S \circ G$ ). Then, given morphisms $f: U \longrightarrow V, g: U^{\prime} \longrightarrow U$ and $h: V \longrightarrow V^{\prime}$ in $\mathbf{M}$, the following diagram commutes (in the category of functors from $\mathbf{N}\left(V^{\prime}\right)$ to $\left.\mathbf{N}\left(U^{\prime}\right)\right)$ :

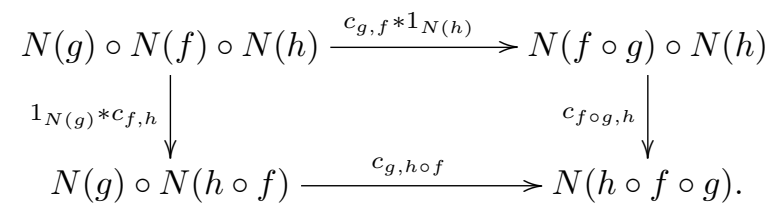


With the clivage $c$ being normalised, we have $\mathbf{N}\left(i d_{U}\right)=i d_{\mathbf{N}(U)}$ for each $U \in \mathbf{M}$. Then, the data (a), (b), (c) given above define a lax 2-functor $N: \mathbf{M}^{o p} \longrightarrow$ Cat (Cat being the 2-category of categories) (see [7, §3.3.2] or [8, Definition 3.10]). Further, it may be shown that there is a one-to-one correspondence between lax 2-functors $N: \mathbf{M}^{o p} \longrightarrow$ Cat and pairs $\underline{\mathbf{N}}=(p: \mathbf{N} \longrightarrow \mathbf{M}, c)$ consisting of a fibred category $p: \mathbf{N} \longrightarrow \mathbf{M}$ with a choice of a normalised clivage $c$ (see $[\mathbf{7}, \S 3.3]$ ).

By a functor $\underline{F}=(\tilde{F}, F)$ of fibred categories from $p: \mathbf{N} \longrightarrow \mathbf{M}$ to $p^{\prime}: \mathbf{N}^{\prime} \longrightarrow \mathbf{M}^{\prime}$, we will mean a functor $\tilde{F}: \mathbf{N} \longrightarrow \mathbf{N}^{\prime}$ such that $\tilde{F}$ descends to a functor $F: \mathbf{M} \longrightarrow \mathbf{M}^{\prime}$ on the base categories, i.e., $p^{\prime} \circ \tilde{F}=F \circ p$. Note that we will not need to assume that $\tilde{F}: \mathbf{N} \longrightarrow \mathbf{N}^{\prime}$ carries cartesian morphisms to cartesian morphisms. We shall give several natural examples of fibred categories when we deal with fibred model categories in Section 3.

\section{Lifting of the model structure}

In this section, we fix a fibred category $p: \mathbf{N} \longrightarrow \mathbf{M}$, with a chosen normalised clivage $c$. Suppose that $\mathbf{M}$ carries a model structure induced by a triple $(\mathbf{C}, \mathbf{W}, \mathbf{F})$, where $\mathbf{C}, \mathbf{W}$ and $\mathbf{F}$ denote respectively the collection of cofibrations, weak equivalences and fibrations for the model structure. For the definition and basic properties of model categories, the reader may see [2, Chapter 1]. Through a series of lemmas, we shall now show that the model structure on $\mathbf{M}$ induces a model structure on $\mathbf{N}$. First, we come to our definition of a fibred model category:

Definition 3.1. Let $p: \mathbf{N} \longrightarrow \mathbf{M}$ be a fibred category and let $\mathbf{M}$ be a model category with the model structure defined by a triple $(\mathbf{C}, \mathbf{W}, \mathbf{F})$. Further, suppose that both $\mathbf{N}$ and $\mathbf{M}$ contain zero objects $0_{N}$ and $0_{M}$ respectively and that $p\left(0_{N}\right)=0_{M}$. On $\mathbf{N}$, define three classes of morphisms:

$\mathbf{C}_{N}$ : Morphisms $\phi$ in $\mathbf{N}$ such that $p(\phi)$ lies in $\mathbf{C}$.

$\mathbf{W}_{N}$ : Morphisms $\phi$ in $\mathbf{N}$ such that $p(\phi)$ lies in $\mathbf{W}$.

$\mathbf{F}_{N}$ : Morphisms $\phi$ in $\mathbf{N}$ such that $p(\phi)$ lies in $\mathbf{F}$ and $\phi$ is cartesian over $p(\phi)$.

We will refer to the triple $(\mathbf{N}, p, \mathbf{M})$ as a fibred model category. A functor

$$
\underline{F}:(\mathbf{N}, p, \mathbf{M}) \longrightarrow\left(\mathbf{N}^{\prime}, p^{\prime}, \mathbf{M}^{\prime}\right)
$$

of fibred model categories is a functor $\underline{F}=(\tilde{F}, F)$ of the underlying fibred categories from $p: \mathbf{N} \longrightarrow \mathbf{M}$ to $p^{\prime}: \mathbf{N}^{\prime} \longrightarrow \mathbf{M}^{\prime}$ as defined in Section 2 .

For the rest of this section, we will continue to use the notation of Definition 3.1. Further, we will assume that $(\alpha, \beta)$ and $(\gamma, \delta)$ are the two functorial factorizations on M. That is, given a morphism $f: U \longrightarrow V$ in $\mathbf{M}$ :

(a) $f=\beta(f) \circ \alpha(f)$, where $\alpha(f)$ is a cofibration and $\beta(f)$ a trivial fibration, and

(b) $f=\delta(f) \circ \gamma(f)$, where $\gamma(f)$ is a trivial cofibration and $\delta(f)$ a fibration.

We will now establish that the triple $\left(\mathbf{C}_{N}, \mathbf{W}_{N}, \mathbf{F}_{N}\right)$ defines a model structure on $\mathbf{N}$. 
Lemma 3.2. Let $(\mathbf{N}, p, \mathbf{M})$ be a fibred model category. Consider the following commutative square in $\mathbf{N}$ :

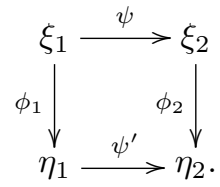

Suppose that $\phi_{1}$ lies in $\mathbf{C}_{N}$ and $\phi_{2}$ lies in $\mathbf{F}_{N}$. Then, if at least one of $\phi_{1}$ and $\phi_{2}$ lies in $\mathbf{W}_{N}$, there exists a morphism $\theta: \eta_{1} \longrightarrow \xi_{2}$ such that $\psi=\theta \circ \phi_{1}$ and $\psi^{\prime}=\phi_{2} \circ \theta$.

Proof. We first consider the case in which $\phi_{1}$ lies in $\mathbf{W}_{N}$. We consider the image (6) of diagram (5) in $\mathbf{M}$ :

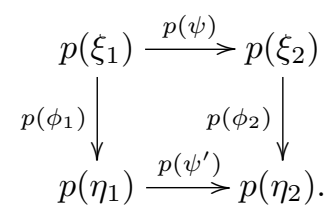

From Definition 3.1, we know that $p\left(\phi_{1}\right)$ lies in both $\mathbf{C}$ and $\mathbf{W}$, while $p\left(\phi_{2}\right)$ lies in $\mathbf{F}$. From the model structure of $\mathbf{M}$, it follows that we have a morphism $s: p\left(\eta_{1}\right) \longrightarrow p\left(\xi_{2}\right)$ such that $p\left(\psi^{\prime}\right)=p\left(\phi_{2}\right) \circ s$ and $p(\psi)=s \circ p\left(\phi_{1}\right)$. Furthermore, since $\phi_{2}$ lies in $\mathbf{F}_{N}$, it follows that $\phi_{2}$ is cartesian over $p\left(\phi_{2}\right)$. The morphism $\psi^{\prime}: \eta_{1} \longrightarrow \eta_{2}$ is such that its image $p\left(\psi^{\prime}\right)$ factors through $p\left(\phi_{2}\right)$ as $p\left(\psi^{\prime}\right)=p\left(\phi_{2}\right) \circ s$. Hence, there exists a morphism $\theta: \eta_{1} \longrightarrow \xi_{2}$ in $\mathbf{N}$ such that $\psi^{\prime}=\phi_{2} \circ \theta$ and $p(\theta)=s$.

It remains to show that $\theta \circ \phi_{1}=\psi$. To do so, we note that $\phi_{2} \circ \psi=\psi^{\prime} \circ \phi_{1}=$ $\phi_{2} \circ\left(\theta \circ \phi_{1}\right)$. Also, $p(\psi)=s \circ p\left(\phi_{1}\right)=p\left(\theta \circ \phi_{1}\right)$. Since $\phi_{2}$ is cartesian over $p\left(\phi_{2}\right)$, it now follows from the uniqueness condition in Definition 2.1 that $\psi=\theta \circ \phi_{1}$.

It is clear that the case in which $\phi_{2}$ lies in $\mathbf{W}_{N}$ can be similarly proved.

Lemma 3.3. Let $\phi: \xi \longrightarrow \eta$ be a morphism in $\mathbf{N}$. Then there exist factorizations $\left(\alpha_{N}, \beta_{N}\right)$ and $\left(\gamma_{N}, \delta_{N}\right)$ of $\phi$ such that

(a) $\phi=\beta_{N}(\phi) \circ \alpha_{N}(\phi), \beta_{N}(\phi)$ lies in both $\mathbf{W}_{N}$ and $\mathbf{F}_{N}$ and $\alpha_{N}(\phi)$ lies in $\mathbf{C}_{N}$.

(b) $\phi=\delta_{N}(\phi) \circ \gamma_{N}(\phi), \delta_{N}(\phi)$ lies $\mathbf{F}_{N}$ and $\gamma_{N}(\phi)$ lies in both $\mathbf{C}_{N}$ and $\mathbf{W}_{N}$.

Further, if we have chosen a clivage $c$ for the fibred category $p: \mathbf{N} \longrightarrow \mathbf{M}$, then the factorizations $\left(\alpha_{N}, \beta_{N}\right)$ and $\left(\gamma_{N}, \delta_{N}\right)$ are functorial.

Proof. Consider the image $p(\phi): p(\xi) \longrightarrow p(\eta)$ of the morphism $\phi$ in $\mathbf{M}$. Since $\mathbf{M}$ has a model structure, it follows that we can write $p(\phi)=\beta(p(\phi)) \circ \alpha(p(\phi))$, where $\beta(p(\phi))$ lies in both $\mathbf{F}$ and $\mathbf{W}$ while $\alpha(p(\phi))$ lies in $\mathbf{C}$.

We now choose a morphism $\beta_{N}(\phi): \xi_{1} \longrightarrow \eta$ cartesian over $\beta(p(\phi))$. Since $p(\phi)=$ $\beta(p(\phi)) \circ \alpha(p(\phi))=p\left(\beta_{N}(\phi)\right) \circ \alpha(p(\phi))$ and $\beta_{N}(\phi)$ is cartesian over $\beta(p(\phi))$, there exists a unique morphism $\alpha_{N}(\phi): \xi \longrightarrow \xi_{1}$ such that

$$
\phi=\beta_{N}(\phi) \circ \alpha_{N}(\phi) \text { and } p\left(\alpha_{N}(\phi)\right)=\alpha(p(\phi)) .
$$

Since $\beta_{N}(\phi)$ is cartesian over $\beta(p(\phi))$ and $\beta(p(\phi))$ lies in both $\mathbf{F}$ and $\mathbf{W}$, it follows that $\beta_{N}(\phi)$ lies in both $\mathbf{F}_{N}$ and $\mathbf{W}_{N}$. Further, since $p\left(\alpha_{N}(\phi)\right)=\alpha(p(\phi))$ and $\alpha(p(\phi))$ lies in $\mathbf{C}$, it follows that $\alpha_{N}(\phi)$ lies in $\mathbf{C}_{N}$. This proves (a). 
Part (b) can be similarly proved. We will now show that the choice of a clivage allows us to choose factorizations in $\mathbf{N}$ functorially. Consider a commutative square in $\mathbf{N}$ :

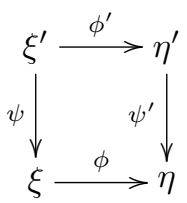

that shows the morphism $\phi^{\prime}$ in $\mathbf{N}$ mapping to the morphism $\phi$ in $\mathbf{N}$. We want to show that the factorization $\beta_{N}\left(\phi^{\prime}\right) \circ \alpha_{N}\left(\phi^{\prime}\right)$ of the morphism $\phi^{\prime}$ maps to the factorization $\beta_{N}(\phi) \circ \alpha_{N}(\phi)$ of the morphism $\phi$. We have the following diagrams:

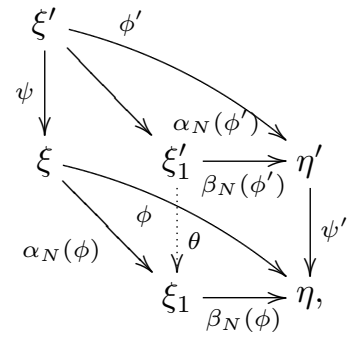

(in $\mathrm{N}$ )

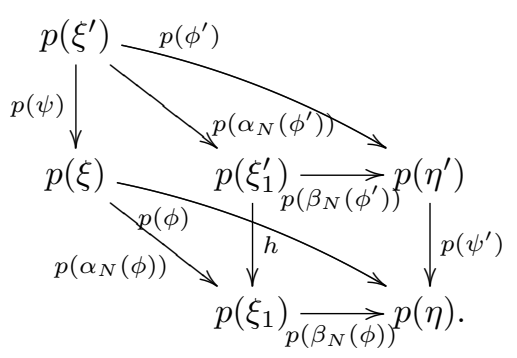

(in $\mathrm{M}$ )

We want to show that the map $\theta: \xi_{1}^{\prime} \longrightarrow \xi_{1}$ exists and makes the diagram in $\mathbf{N}$ commutative. We have already defined $\alpha_{N}$ and $\beta_{N}$ in such a way that

$$
\begin{array}{ll}
p\left(\alpha_{N}(\phi)\right)=\alpha(p(\phi)) & p\left(\alpha_{N}\left(\phi^{\prime}\right)\right)=\alpha\left(p\left(\phi^{\prime}\right)\right), \\
p\left(\beta_{N}(\phi)\right)=\beta(p(\phi)) & p\left(\beta_{N}\left(\phi^{\prime}\right)\right)=\beta\left(p\left(\phi^{\prime}\right)\right) .
\end{array}
$$

The map $h: p\left(\xi_{1}^{\prime}\right) \longrightarrow p\left(\xi_{1}\right)$ exists and makes the diagram in $\mathbf{M}$ commutative because $(\alpha, \beta)$ is a functorial factorization of morphisms of $\mathbf{M}$.

Now, given the chosen clivage $c$, we have $\beta_{N}(\phi):=c_{\beta(p(\phi))}(\eta): C=\beta(p(\phi))^{*}(\eta)$ $\longrightarrow \eta$ and $\beta_{N}\left(\phi^{\prime}\right):=c_{\beta\left(p\left(\phi^{\prime}\right)\right)}\left(\eta^{\prime}\right): \xi_{1}^{\prime}=\beta\left(p\left(\phi^{\prime}\right)\right)^{*}\left(\eta^{\prime}\right) \longrightarrow \eta^{\prime}$. Then, from the discussion following diagram (4) in Section 2.1, we note that there must exist $\theta: \xi_{1}^{\prime}=$ $\beta\left(p\left(\phi^{\prime}\right)\right)^{*}\left(\eta^{\prime}\right) \longrightarrow \beta(p(\phi))^{*}(\eta)=\xi_{1}$ such that $\psi^{\prime} \circ \beta_{N}\left(\phi^{\prime}\right)=\beta_{N}(\phi) \circ \theta$.

It remains to show that $\theta \circ \alpha_{N}\left(\phi^{\prime}\right)=\alpha_{N}(\phi) \circ \psi$. To prove this, we consider the morphism $\psi^{\prime} \circ \phi^{\prime}: \xi^{\prime} \longrightarrow \eta$. We note that

$$
\begin{gathered}
\psi^{\prime} \circ \phi^{\prime}=\psi^{\prime} \circ\left(\beta_{N}\left(\phi^{\prime}\right) \circ \alpha_{N}\left(\phi^{\prime}\right)\right)=\beta_{N}(\phi) \circ\left(\theta \circ \alpha_{N}\left(\phi^{\prime}\right)\right), \\
\psi^{\prime} \circ \phi^{\prime}=\phi \circ \psi=\beta_{N}(\phi) \circ\left(\alpha_{N}(\phi) \circ \psi\right),
\end{gathered}
$$

and, further,

$$
p\left(\theta \circ \alpha_{N}\left(\phi^{\prime}\right)\right)=h \circ \alpha\left(p\left(\phi^{\prime}\right)\right)=\alpha(p(\phi)) \circ p(\psi)=p\left(\alpha_{N}(\phi) \circ \psi\right) .
$$

Since $\beta_{N}(\phi)$ is cartesian over its image $\beta(p(\phi))$ in $\mathbf{M}$, it follows from the uniqueness condition in Definition 2.1 that $\left(\theta \circ \alpha_{N}\left(\phi^{\prime}\right)\right)=\left(\alpha_{N}(\phi) \circ \psi\right)$. Similarly, we can show that the factorization $\left(\gamma_{N}, \delta_{N}\right)$ is also functorial on the morphisms of $\mathbf{N}$.

We now have all we need to prove the following theorem. 
Theorem 3.4. Let $\mathbf{M}$ be a model category and let $p: \mathbf{N} \longrightarrow \mathbf{M}$ be a category fibred over $\mathbf{M}$ with a chosen normalised clivage $c$. Then, the triple $\left(\mathbf{C}_{N}, \mathbf{W}_{N}, \mathbf{F}_{N}\right)$, as defined in Definition 3.1, determines a model structure on $\mathbf{N}$.

Proof. For two given (composable) morphisms $\phi_{1}$ and $\phi_{2}$ in $\mathbf{N}$, suppose that two out of the three morphisms $\phi_{1}, \phi_{2}$ and $\phi_{1} \circ \phi_{2}$ lie in $\mathbf{W}_{N}$. Then, it follows that two out of the three morphisms: $p\left(\phi_{1}\right), p\left(\phi_{2}\right)$ and $p\left(\phi_{1} \circ \phi_{2}\right)$ lie in $\mathbf{W}$, and hence so does the third. From Definition 3.1, it follows that $\mathbf{W}_{N}$ satisfies the two-out-of-three property.

Further, suppose that $\phi$ and $\phi^{\prime}$ are two morphisms in $\mathbf{N}$ such that $\phi^{\prime}$ is a retract of $\phi$. Then, $p\left(\phi^{\prime}\right)$ is a retract of $p(\phi)$ in $\mathbf{M}$. Using the model structure on $\mathbf{M}$, it follows that if $p(\phi)$ lies in $\mathbf{C}, \mathbf{W}$ or $\mathbf{F}$ resp., then so does $p\left(\phi^{\prime}\right)$. Hence, $\phi \in \mathbf{C}_{N}$ implies that $\phi^{\prime} \in \mathbf{C}_{N}$ and $\phi \in \mathbf{W}_{N}$ implies that $\phi^{\prime} \in \mathbf{W}_{N}$. From Lemma 2.3 it follows that if $\phi$ is cartesian over $p(\phi)$, then $\phi^{\prime}$ is cartesian over $p\left(\phi^{\prime}\right)$. Hence, $\phi \in \mathbf{F}_{N}$ implies $\phi^{\prime} \in \mathbf{F}_{N}$.

The two lifting properties that are required in order for $\left(\mathbf{C}_{N}, \mathbf{W}_{N}, \mathbf{F}_{N}\right)$ to define a model structure on $\mathbf{N}$ follow directly from Lemma 3.2. Finally, Lemma 3.3 shows that the chosen normalised clivage $c$ determines functorial factorizations in $\mathbf{N}$. This proves the theorem.

Remark 3.5.

(1) Henceforth, whenever we say that $(\mathbf{N}, p, \mathbf{M})$ is a fibred model category, it is understood that $\mathbf{N}$ carries the model structure $\left(\mathbf{C}_{N}, \mathbf{W}_{N}, \mathbf{F}_{N}\right)$ lifted from $\mathbf{M}$. We also imply that the underlying fibred category $p: \mathbf{N} \longrightarrow \mathbf{M}$ has a chosen clivage $c$. In Definition 3.1, we have assumed that both $\mathbf{N}$ and $\mathbf{M}$ contain zero objects $0_{N}$ and $0_{M}$ respectively, but we will drop the subscripts and denote both zero objects simply by 0 .

(2) In the modern definition of a model category, one usually also assumes that the category contains all small limits and colimits. If the reader so desires, he may add the assumption that " $\mathbf{N}$ contains all small limits and colimits and $p$ commutes with small limits and colimits" to the definition of a fibred model category $(\mathbf{N}, p, \mathbf{M})$ in Definition 3.1. The condition that $p$ commutes with small colimits will be very important to us in Section 4 .

We make note of the following result on cofibrant and fibrant replacement functors: We recall that the cofibrant replacement (resp. fibrant replacement) $Q U \stackrel{q}{\longrightarrow} U$ (resp. $U \stackrel{r}{\longrightarrow} R U$ ) of an object $U$ of a model category $\mathbf{M}$ is obtained by factorizing the map $0 \longrightarrow U$ (resp. $U \longrightarrow 0$ ) into a cofibration $0 \longrightarrow Q U$ (resp. a fibration $R U \longrightarrow 0$ ) and a trivial fibration $Q U \stackrel{q}{\longrightarrow} U$ (resp. a trivial cofibration $U \stackrel{r}{\longrightarrow} R U$ ).

Corollary 3.6. Let $(\mathbf{N}, p, \mathbf{M})$ be a fibred model category. Let $Q_{N}$ and $Q_{M}$ denote the cofibrant replacement functors on the categories $\mathbf{N}$ and $\mathbf{M}$ respectively, with $R_{N}$ and $R_{M}$ denoting their respective fibrant replacement functors. Then, given an object $\xi$ of $\mathbf{N}$, we have

$$
p\left(Q_{N} \xi\right)=Q_{M} p(\xi), \quad p\left(R_{N} \xi\right)=R_{M} p(\xi) .
$$

Proof. Considering the morphisms $0 \longrightarrow \xi$ and $\xi \longrightarrow 0$ in $\mathbf{N}$, the result is clear from the proof of Lemma 3.3.

We will now show that, under certain conditions, the lifted model structure on $\mathbf{N}$ can be used to lift right homotopic maps from $\mathbf{M}$ to $\mathbf{N}$. Recall that a path object 
for some $X \in \mathbf{M}$ is a factorization of the diagonal map $\Delta_{X}: X \longrightarrow X \times X$ into a weak equivalence $s: X \longrightarrow X^{\prime}$ followed by a fibration $\left(p_{0}, p_{1}\right): X^{\prime} \longrightarrow X \times X$. Given two maps $f, g: B \longrightarrow X$ in $\mathbf{M}$, a right homotopy from $f$ to $g$ consists of a map $K: B \longrightarrow X^{\prime}$ for some path object $X^{\prime}$ of $X$ such that $p_{0} K=f$ and $p_{1} K=g$.

Suppose that the category $\mathbf{N}$ contains finite products. Given the fibred category $p: \mathbf{N} \longrightarrow \mathbf{M}$, we will say that $p$ commutes with coordinate projections if, given any $\xi, \xi^{\prime} \in \mathbf{N}$ and the coordinate projections $\pi_{0}: \xi \times \xi^{\prime} \longrightarrow \xi, \pi_{1}: \xi \times \xi^{\prime} \longrightarrow \xi^{\prime}$, we have $p\left(\xi \times \xi^{\prime}\right)=p(\xi) \times p\left(\xi^{\prime}\right)$ and $p\left(\pi_{0}\right): p(\xi) \times p\left(\xi^{\prime}\right) \longrightarrow p(\xi), p\left(\pi_{1}\right): p(\xi) \times p\left(\xi^{\prime}\right) \longrightarrow$ $p\left(\xi^{\prime}\right)$ are also coordinate projections.

Proposition 3.7. Let $p: \mathbf{N} \longrightarrow \mathbf{M}$ be a fibred model category. Suppose that the category $\mathbf{N}$ has finite products and that $p: \mathbf{N} \longrightarrow \mathbf{M}$ commutes with coordinate projections. Then, given right homotopic maps $f, g: U \longrightarrow V$ in $\mathbf{M}$ and some $\eta \in \mathbf{N}$ such that $p(\eta)=V$, there exist right homotopic maps $\phi, \psi: \xi \longrightarrow \eta$ in $\mathbf{N}$ such that $p(\phi)=f$ and $p(\psi)=g$.

Proof. The maps $f, g: B \longrightarrow X$ induce a single map $(f, g): B \longrightarrow X \times X$. Since N contains finite products and $p$ commutes with coordinate projections, $p(\eta \times \eta)=X$ $\times X$. We choose a morphism $(\phi, \psi): \xi \longrightarrow \eta \times \eta$ in $\mathbf{N}$ cartesian over $(f, g): B \longrightarrow$ $X \times X$. Let $\pi_{0}: \eta \times \eta \longrightarrow \eta$ and $\pi_{1}: \eta \times \eta \longrightarrow \eta$ be the two coordinate projections. We set $\phi=\pi_{0} \circ(\phi, \psi)$ and $\psi=\pi_{1} \circ(\phi, \psi)$. Then, $p(\phi)=f$ and $p(\psi)=g$.

Since $f$ is homotopic to $g$, it follows from the definitions that there exists a path object $X^{\prime}$ for $X$ and a map $K: B \longrightarrow X^{\prime}$ such that $p_{0} K=f$ and $p_{1} K=g$. Suppose that the path object $X^{\prime}$ is obtained by factorizing the diagonal morphism $\Delta_{X}: X \longrightarrow$ $X \times X$ into a weak equivalence $s: X \longrightarrow X^{\prime}$ followed by a fibration $\left(p_{0}, p_{1}\right): X^{\prime} \longrightarrow$ $X \times X$.

Consider the diagonal morphism $\Delta_{\eta}: \eta \longrightarrow \eta \times \eta$ and a morphism $\Phi: \eta^{\prime} \longrightarrow \eta \times \eta$ in $\mathbf{N}$ cartesian over $\left(p_{0}, p_{1}\right): X^{\prime} \longrightarrow X \times X$. Since $\left(p_{0}, p_{1}\right)$ is a fibration in $\mathbf{M}, \Phi$ is a fibration in $\mathbf{N}$. Also, $p(\Phi)=\left(p_{0}, p_{1}\right) \circ s$. Since $\Phi: \eta^{\prime} \longrightarrow \eta \times \eta$ is cartesian over $\left(p_{0}, p_{1}\right)$, there exists a unique $\Psi: \eta \longrightarrow \eta^{\prime}$ such that $p(\Psi)=s$ and $\Delta_{\eta}=\Phi \circ \Psi$. Then, $\Psi$ is a weak equivalence, and hence $\eta^{\prime}$ is a path object for $\eta$.

Since $\Phi: \eta^{\prime} \longrightarrow \eta \times \eta$ is cartesian over $\left(p_{0}, p_{1}\right)$ and $p(\phi, \psi)=(f, g)=\left(p_{0}, p_{1}\right) \circ K=$ $p(\Phi) \circ K$, there exists a unique $\theta: \xi \longrightarrow \eta^{\prime}$ such that $(\phi, \psi)=\Phi \circ \theta$ and $p(\theta)=K$. Then, $\phi=\left(\pi_{0} \circ \Phi\right) \circ \theta$ and $\psi=\left(\pi_{1} \circ \Phi\right) \circ \theta$. This shows that $\phi$ is right homotopic to $\psi$.

We end this section by giving several examples of lifted model structures.

(1) Let $\mathbf{M}$ be a model category and let $\operatorname{Mor}(\mathbf{M})$ be the category of morphisms of $\mathbf{M}$. We define the functor $p$ : $\operatorname{Mor}(\mathbf{M}) \longrightarrow \mathbf{M}$ by taking a morphism $f: U \longrightarrow V$ of objects in $\mathbf{M}$ (then $f \in \operatorname{Mor}(\mathbf{M})$ ) to its target $V$. Then, if the category $\mathbf{M}$ contains fibre products, it can be shown (see $[\mathbf{7}, \S 3.1 .9]$ ) that $\operatorname{Mor}(\mathbf{M})$ is a fibred category over M. From Theorem 3.4, it follows that if $\mathbf{M}$ is a model category containing fibre products, then $\operatorname{Mor}(\mathbf{M})$ carries a model structure.

(2) Let $\mathbf{M}$ be the model category Top of topological spaces with the morphisms being continuous maps. For some $n \geqslant 1$, one could let $\mathbf{N}:=\mathbf{V B}_{n}$, the category of $n$-dimensional (say complex) vector bundles over topological spaces. Then, for each object $X$ of Top, we have a category $\mathbf{V B}_{n}(X)$ of $n$-dimensional vector bundles over $X$. Further, for each morphism $f: X \longrightarrow Y$ in Top, we can choose a pullback functor 
$f^{*}: \mathbf{V B}_{n}(Y) \longrightarrow \mathbf{V B}_{n}(X)$ with $i d_{Y}^{*}=i d_{\mathbf{V B}_{n}(Y)}$. We have isomorphisms of functors $g^{*} \circ f^{*} \cong(f \circ g)^{*}$ for every pair of composable morphisms $f, g$. These isomorphisms are natural, and, from the discussion at the end of Section 2, it now follows that the category $\mathbf{V B}_{n}$ is fibred over the category Top. This defines a natural lifted model structure on the category $\mathbf{V B}_{n}$ for each $n \geqslant 1$.

(3) Given a category $\mathbf{C}$, denote by $\hat{\mathbf{C}}:=\operatorname{Func}\left(\mathbf{C}^{o p}\right.$, Sets $)$ the category of contravariant functors from $\mathbf{C}$ to the category Sets of sets, i.e., the category of presheaves of sets on $\mathbf{C}$. For any object $X$ in $\mathbf{C}$, let us denote by $h_{X}$ the presheaf on $\mathbf{C}$ represented by $X$. Then, to each object $X$ in $\mathbf{C}$, we can associate the category $\hat{\mathbf{C}} / h_{X}$ of objects in $\hat{\mathbf{C}}$ over $h_{X}$. Since the category $\hat{\mathbf{C}}$ always contains fibre products, given any morphism $f: X \longrightarrow Y$ in $\mathbf{C}$, we have a corresponding pullback functor $f^{*}: \hat{\mathbf{C}} / h_{Y} \longrightarrow \hat{\mathbf{C}} / h_{X}$. Then, it follows from $[\mathbf{7}, \S 3.1 .10]$ that this data determines a fibred category $\operatorname{Psh}(\mathbf{C})$ over $\mathbf{C}$. For instance, let $\mathbf{C}$ be the category Top of topological spaces with morphisms being continuous maps. Then, by the same reasoning as above, the model structure on Top lifts to a model structure on the category $\mathbf{P s h}(\mathbf{T o p})$ fibred over Top.

(4) Let $\mathbf{M}$ be a model category and let $U$ be an object of $\mathbf{M}$. Then, the objects of the category $\mathbf{M} / U$ are morphisms $f: V \longrightarrow U$ of $\mathbf{M}$. A morphism from $f: V \longrightarrow U$ to $f^{\prime}: V^{\prime} \longrightarrow U$ in $\mathbf{M} / U$ consists of a morphism $g: V \longrightarrow V^{\prime}$ in $\mathbf{M}$ such that $f=f^{\prime} \circ g$.

We may see the category $\mathbf{M} / U$ as a category fibred over $\mathbf{M}$ in the following manner: Let $h_{U}$ denote the presheaf on $\mathbf{M}$ represented by $U$, i.e., the presheaf defined by taking $h_{U}(V)=\operatorname{Mor}_{\mathbf{M}}(V, U)$ for each object $V$ of $\mathbf{M}$. Then, to the presheaf $h_{U}$ of sets, we can associate the category $\mathbf{N}$ whose objects are pairs $(V, f)$ with $V \in \mathbf{M}$ and $f$ a morphism $f: V \longrightarrow U$. Then, a morphism $\phi:(V, f) \longrightarrow\left(V^{\prime}, f^{\prime}\right)$ in $\mathbf{N}$ is given by a morphism $g: V \longrightarrow V^{\prime}$ in $\mathbf{M}$ such that $f=f^{\prime} \circ g$. Then, the functor $p: \mathbf{N} \longrightarrow \mathbf{M}$ defined by $p(V, f)=V$ for each $(V, f) \in \mathbf{N}$ makes $\mathbf{N}$ into a fibred category over $\mathbf{M}$.

Note that the category $\mathbf{N}$ is identical to $\mathbf{M} / U$. Then, Theorem 3.4 shows that $\mathbf{M} / U$ carries a model structure, which can be described as follows: A morphism $g:(V, f) \longrightarrow\left(V^{\prime}, f^{\prime}\right)$ is a cofibration, weak equivalence or fibration in $\mathbf{M} / U$ according as $g: V \longrightarrow V^{\prime}$ is a cofibration, weak equivalence or fibration in $\mathbf{M}$.

(5) Suppose that $\mathbf{M}$ is a 2-category whose underlying 1-category is a model category. Then, for any two objects $U, V \in \mathbf{M}$, we have a category $\operatorname{Mor}(U, V)$ of 1-morphisms from $U$ to $V$. A 1-morphism $f: U \longrightarrow U^{\prime}$ in $\mathbf{M}$ induces a functor $f^{*}: \operatorname{Mor}\left(U^{\prime}, V\right) \longrightarrow \operatorname{Mor}(U, V)$. It follows that, for each object $V$, we can define a fibred category $\mathbf{M} / V$ as follows:

(a) For each $U \in \mathbf{M}$, we set $(\mathbf{M} / V)(U):=\operatorname{Mor}(U, V)$.

(b) For each 1-morphism $f: U \longrightarrow U^{\prime}$, we have an obvious functor $(\mathbf{M} / V)(f)=$ $f^{*}: \operatorname{Mor}\left(U^{\prime}, V\right) \longrightarrow \operatorname{Mor}(U, V)$.

(c) Given composable 1-morphisms $f: U \longrightarrow U^{\prime}$ and $g: U^{\prime} \longrightarrow U^{\prime \prime}$ in $\mathbf{M}$, it follows from the definition of a 2-category that there are natural isomorphisms $c_{f, g}$ of functors $f^{*} \circ g^{*} \cong(g \circ f)^{*}: \operatorname{Mor}\left(U^{\prime \prime}, V\right) \longrightarrow \operatorname{Mor}(U, V)$.

Then, from the discussion at the end of Section 2, it follows that (a), (b) and (c) determine a fibred category $\mathbf{M} / V$ over $\mathbf{M}$ (with a chosen normalised clivage). Then, Theorem 3.4 shows that the category $\mathbf{M} / V$ carries a model structure.

(6) Let $\mathbf{C}^{\tau}=(\mathbf{C}, \tau)$ be a site with underlying category $\mathbf{C}$ and Grothendieck topology $\tau$. Consider the category $\operatorname{Cov}^{\tau}(\mathbf{C})$ of $\tau$-coverings of objects of $\mathbf{C}$, defined as follows: The objects of the category $\operatorname{Cov}^{\tau}(\mathbf{C})$ are of the form $\left(U,\left\{p_{i}: U_{i} \longrightarrow U\right\}_{i \in I}\right)$, 
where $\left\{p_{i}: U_{i} \longrightarrow U\right\}_{i \in I}$ is a $\tau$-covering of the object $U \in \mathbf{C}$. A morphism $F=$ $\left(f, f_{i}\right):\left(U^{\prime},\left\{p_{i}^{\prime}: U_{i}^{\prime} \longrightarrow U^{\prime}\right\}_{i \in I}\right) \longrightarrow\left(U,\left\{p_{i}: U_{i} \longrightarrow U\right\}_{i \in I}\right)$ consists of a morphism $f: U^{\prime} \longrightarrow U$ and fibre squares

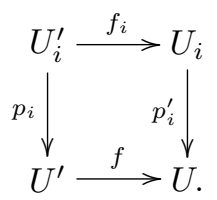

The obvious functor $p^{\tau}: \operatorname{Cov}^{\tau}(\mathbf{C}) \longrightarrow \mathbf{C}$ that takes $\left(U,\left\{p_{i}: U_{i} \longrightarrow U\right\}_{i \in I}\right)$ to $U$ makes $\operatorname{Cov}^{\tau}(\mathbf{C})$ into a category fibred over $\mathbf{C}$, where the morphism

$$
F=\left(f, f_{i}\right):\left(U^{\prime},\left\{p_{i}^{\prime}: U_{i}^{\prime} \longrightarrow U^{\prime}\right\}_{i \in I}\right) \longrightarrow\left(U,\left\{p_{i}: U_{i} \longrightarrow U\right\}_{i \in I}\right)
$$

is cartesian over the morphism $f$ in $\mathbf{C}$. Now, if the underlying category $\mathbf{C}$ is a model category, then it follows that $\operatorname{Cov}^{\tau}(\mathbf{C})$ carries a lifted model structure. For instance, this shows that the "category of open covers of topological spaces", or the "category of étale covers of topological spaces" carries a model structure. Recall here that an étale cover $\left\{\pi_{i}: U_{i} \longrightarrow U\right\}_{i \in I}$ of a topological space $U$ is a jointly surjective family of local homeomorphisms $\pi_{i}: U_{i} \longrightarrow U, i \in I$.

(7) This example is a refinement of Example (1). Let $\mathbf{M}$ be a model category that contains fibre products and let $P$ be a property of morphisms in $\mathbf{M}$ that is stable under base change. That is, if the morphism $f: U \longrightarrow V$ in $\mathbf{M}$ has property $P, g: V^{\prime} \longrightarrow V$ is any morphism in $\mathbf{M}$, and we consider the fibre square

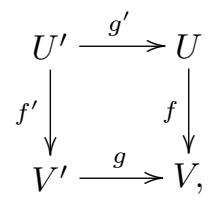

then the morphism $f^{\prime}: U^{\prime} \longrightarrow V^{\prime}$ also has property $P$. For instance, $\mathbf{M}$ could be the category of topological spaces, and $P$ could be the property of a morphism being an open immersion (or being proper, or étale, or surjective, etc.). Then, denote by $\operatorname{Mor}^{P}(\mathbf{M})$ the category of morphisms of $\mathbf{M}$ having property $P$. A morphism $G=$ $\left(g_{1}, g_{2}\right):\left(f^{\prime}: U^{\prime} \longrightarrow V^{\prime}\right) \longrightarrow(f: U \longrightarrow V)$ in $\operatorname{Mor}^{P}(\mathbf{M})$ is given by a commutative square

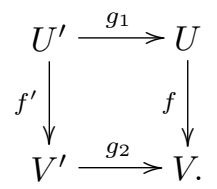

The obvious morphism that takes $(f: U \longrightarrow V) \in \operatorname{Mor}^{P}(\mathbf{M})$ to $V$ makes $\operatorname{Mor}^{P}(\mathbf{M})$ into a fibred category over $\mathbf{M}$, where the morphism $G=\left(g_{1}, g_{2}\right):\left(f^{\prime}: U^{\prime} \longrightarrow V^{\prime}\right) \longrightarrow$ $(f: U \longrightarrow V)$ is cartesian over $g_{2}: V^{\prime} \longrightarrow V$ if diagram (7) is a fibre square. Then, it follows that $\operatorname{Mor}^{P}(\mathbf{M})$ carries a lifted model structure. 


\section{Monoidal fibred model categories and Quillen adjunctions}

In the previous section, we have shown how the model category structure can be lifted from a base category $\mathbf{M}$ to a category $\mathbf{N}$ fibred over $\mathbf{M}$. In this section, we will consider monoidal structures and Quillen pairs on fibred model categories. We will continue to use the notation defined in Sections 2 and 3. Whenever we talk about a fibred category $p: \mathbf{N} \longrightarrow \mathbf{M}$, it will be understood that we also have a chosen normalised clivage $c$.

We start with Quillen adjunctions. The following result shows that the lifted model structures behave well with respect to Quillen adjunctions and equivalences.

Proposition 4.1. Let $(\mathbf{N}, p, \mathbf{M})$ and $\left(\mathbf{N}^{\prime}, p^{\prime}, \mathbf{M}^{\prime}\right)$ be two fibred model categories and let $(\underline{F}, \underline{G})$ be a pair of functors of fibred model categories, $\underline{F}=(\tilde{F}, F):(\mathbf{N}, p, \mathbf{M}) \longrightarrow$ $\left(\mathbf{N}^{\prime}, p^{\prime}, \mathbf{M}^{\prime}\right), \underline{G}=(\tilde{G}, G):\left(\mathbf{N}^{\prime}, p^{\prime}, \mathbf{M}^{\prime}\right) \longrightarrow(\mathbf{N}, p, \mathbf{M})$ such that

(1) $\tilde{F}: \mathbf{N} \longrightarrow \mathbf{N}^{\prime}$ is left adjoint to $\tilde{G}: \mathbf{N}^{\prime} \longrightarrow \mathbf{N}$.

(2) $F: \mathbf{M} \longrightarrow \mathbf{M}^{\prime}$ is left adjoint to $G: \mathbf{M}^{\prime} \longrightarrow \mathbf{M}$ and $(F, G)$ is a Quillen adjunction of model categories $\mathbf{M}$ and $\mathbf{M}^{\prime}$.

Then the pair $(\tilde{F}, \tilde{G})$ is a Quillen adjunction of model categories $\mathbf{N}$ and $\mathbf{N}^{\prime}$, the model structures on $\mathbf{N}$ and $\mathbf{N}^{\prime}$ being those lifted from $\mathbf{M}$ and $\mathbf{M}^{\prime}$ respectively. Further, if $(F, G)$ is a Quillen equivalence, then so is $(\tilde{F}, \tilde{G})$.

Proof. Let $\phi: \xi_{1} \longrightarrow \xi_{2}$ be a morphism in $\mathbf{N}$. If $\phi$ is a cofibration (resp. trivial cofibration) in $\mathbf{N}$, then $p(\phi)$ is a cofibration (resp. trivial cofibration) in $\mathbf{M}$. Since $F$ is a left Quillen functor, $p^{\prime}(\tilde{F}(\phi))=F(p(\phi))$ is a cofibration (resp. trivial cofibration) in $\mathbf{M}^{\prime}$. Hence, $\tilde{F}(\phi)$ is a cofibration (resp. trivial cofibration) in $\mathbf{N}^{\prime}$. Thus, the left adjoint functor $\tilde{F}$ preserves cofibrations and trivial cofibrations, and hence $\tilde{F}$ is a left Quillen functor. Since $\tilde{F}$ and $\tilde{G}$ are adjoint functors, this implies that $\tilde{G}$ is a right Quillen functor (see, for instance, [2, Lemma 1.3.4]), and the pair $(\tilde{F}, \tilde{G})$ defines a Quillen adjunction.

Now suppose that $(F, G)$ is a Quillen equivalence. Then, a morphism $f$ in $\mathbf{M}$ is a weak equivalence if and only if $F(f)$ is a weak equivalence in $\mathbf{M}^{\prime}$. Given a weak equivalence $\phi$ in $\mathbf{N}$, we have $p^{\prime}(\tilde{F}(\phi))=F(p(\phi))$. Since $p(\phi)$ is a weak equivalence in $\mathbf{M}$ and $F$ preserves weak equivalences, it follows that $p^{\prime}(\tilde{F}(\phi))$ is a weak equivalence in $\mathbf{M}^{\prime}$. Thus, by the definition of the lifted model structure on $\mathbf{N}^{\prime}$, it follows that $\tilde{F}(\phi)$ is a weak equivalence in $\mathbf{N}^{\prime}$.

Conversely, suppose that $\phi$ is a morphism in $\mathbf{N}$ such that $\tilde{F}(\phi)$ is a weak equivalence in $\mathbf{N}^{\prime}$. Then $F(p(\phi))=p^{\prime}(\tilde{F}(\phi))$ is a weak equivalence in $\mathbf{M}^{\prime}$. Since $(F, G)$ is a pair of adjoint functors defining a Quillen equivalence, it follows that $p(\phi)$ is a weak equivalence. Again, by the definition of the lifted model structure on $\mathbf{N}$, it follows that $\phi$ is a weak equivalence in $\mathbf{N}$. Thus, if $(F, G)$ is a pair of adjoint functors defining a Quillen equivalence, then $\tilde{F}(\phi)$ is a weak equivalence if and only $\phi$ is. This shows that $(\tilde{F}, \tilde{G})$ itself is a Quillen equivalence.

For instance, suppose that $\mathbf{M}$ and $\mathbf{M}^{\prime}$ are two model categories and let $F: \mathbf{M} \longrightarrow$ $\mathbf{M}^{\prime}$ be a functor left adjoint to $G: \mathbf{M}^{\prime} \longrightarrow \mathbf{M}$ defining a Quillen adjunction of model categories $\mathbf{M}$ and $\mathbf{M}^{\prime}$. Suppose that we can choose a pair of objects $U \in \mathbf{M}$ and $U^{\prime} \in \mathbf{M}^{\prime}$ such that 
(A1) $F U=U^{\prime}$ and $G U^{\prime}=U$.

(A2) The unit and counit maps corresponding to the adjoint pair $(F, G)$ are identities on $U$ and $U^{\prime}$, i.e., the canonical maps $U \rightarrow G F(U)=U$ and $U^{\prime}=F G\left(U^{\prime}\right) \longrightarrow$ $U^{\prime}$ induced by the adjoint pair $(F, G)$ are identities.

Then, it follows that $F: \mathbf{M} \longrightarrow \mathbf{M}^{\prime}$ induces a functor $F / U: \mathbf{M} / U \longrightarrow \mathbf{M}^{\prime} / U^{\prime}$ and $G: \mathbf{M}^{\prime} \longrightarrow \mathbf{M}$ induces a functor $G / U^{\prime}: \mathbf{M}^{\prime} / U^{\prime} \longrightarrow \mathbf{M} / U$.

Lemma 4.2. Let the model categories $\mathbf{M}, \mathbf{M}^{\prime}$, the adjoint functors $F: \mathbf{M} \longrightarrow \mathbf{M}^{\prime}$, $G: \mathbf{M}^{\prime} \longrightarrow \mathbf{M}$, the objects $U \in \mathbf{M}, U^{\prime} \in \mathbf{M}^{\prime}$ and the induced functors $F / U: \mathbf{M} / U \longrightarrow$ $\mathbf{M}^{\prime} / U^{\prime}, G / U^{\prime}: \mathbf{M}^{\prime} / U^{\prime} \longrightarrow \mathbf{M} / U$ be as above. Then, the functors $F / U$ and $G / U^{\prime}$ are adjoint to each other.

Proof. Consider an object $S_{f}=(f: S \longrightarrow U) \in \mathbf{M} / U$ and an object $T_{g}=(g: T \longrightarrow$ $\left.U^{\prime}\right) \in \mathbf{M}^{\prime} / U^{\prime}$. Let $\tilde{\varphi}:(F / U)\left(S_{f}\right) \longrightarrow T_{g}$ be a morphism in $\mathbf{M}^{\prime} / U^{\prime}$. Then $\tilde{\varphi}$ is induced by a morphism $\varphi: F S \longrightarrow T$ in $\mathbf{M}^{\prime}$ such that $g \circ \varphi=F(f)$. By adjointness of $F$ and $G$, the following diagram commutes:

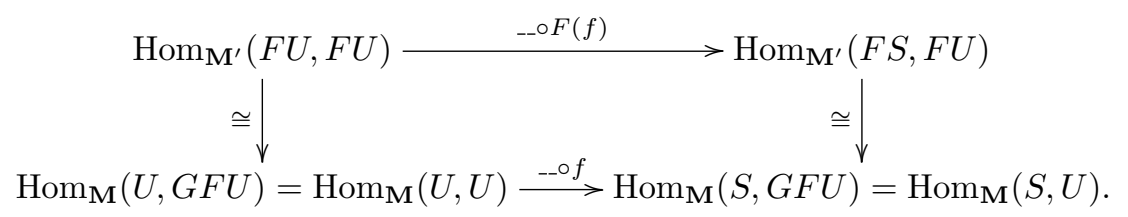

By assumption (A2) above, the left vertical isomorphism in (8) carries $i d_{F(U)} \in$ $\operatorname{Hom}_{\mathbf{M}^{\prime}}(F U, F U)$ to $i d_{U} \in \operatorname{Hom}_{\mathbf{M}}(U, U)$. It follows that the right vertical isomorphism in (8) carries $F(f)=i d_{F(U)} \circ F(f) \in \operatorname{Hom}_{\mathbf{M}^{\prime}}(F S, F U)$ to the map $f=i d_{U} \circ$ $f \in \operatorname{Hom}_{\mathbf{M}}(S, U)$. Again, by adjointness of $F$ and $G$, the following diagram commutes:

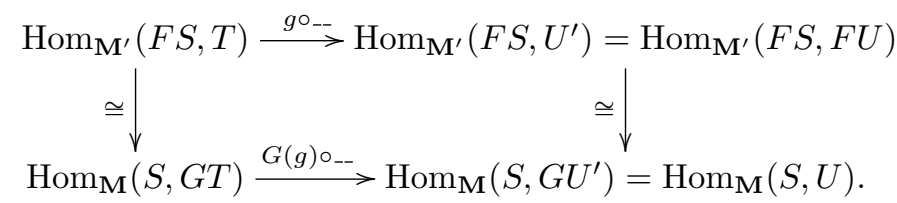

Suppose that the left vertical isomorphism in (9) carries $\varphi \in \operatorname{Hom}_{\mathbf{M}^{\prime}}(F S, T)$ to $\varphi^{\prime} \in$ $\operatorname{Hom}_{\mathbf{M}}(S, G T)$. We know that $g \circ \varphi=F(f) \in \operatorname{Hom}_{\mathbf{M}^{\prime}}\left(F S, U^{\prime}\right)=\operatorname{Hom}_{\mathbf{M}^{\prime}}(F S, F U)$. The right vertical isomorphism in (9) is identical to the right vertical isomorphism in (8), and hence it carries $F(f) \in \operatorname{Hom}_{\mathbf{M}^{\prime}}\left(F S, U^{\prime}\right)=\operatorname{Hom}_{\mathbf{M}^{\prime}}(F S, F U)$ to the map $f \in \operatorname{Hom}_{\mathbf{M}}\left(S, G U^{\prime}\right)=\operatorname{Hom}_{\mathbf{M}}(S, U)$. By commutativity of (9), it now follows that $G(g) \circ \varphi^{\prime}=f$. Hence, $\varphi^{\prime}$ induces a morphism $\tilde{\varphi}^{\prime}: S_{f} \longrightarrow\left(G / U^{\prime}\right)\left(T_{g}\right)$.

Reversing the vertical isomorphisms in (8) and (9), the morphism $\tilde{\varphi}^{\prime}: S_{f} \longrightarrow$ $\left(G / U^{\prime}\right)\left(T_{g}\right)$ corresponds to $\tilde{\varphi}:(F / U)\left(S_{f}\right) \longrightarrow T_{g}$. This shows that there are natural bijections

$$
\operatorname{Hom}_{\mathbf{M}^{\prime} / U^{\prime}}\left((F / U)\left(S_{f}\right), T_{g}\right) \cong \operatorname{Hom}_{\mathbf{M} / U}\left(S_{f},\left(G / U^{\prime}\right)\left(T_{g}\right)\right),
$$

which shows that the functors $F / U$ and $G / U^{\prime}$ are mutually adjoint.

Proposition 4.3. Let the model categories $\mathbf{M}, \mathbf{M}^{\prime}$, the adjoint functors $F: \mathbf{M} \longrightarrow$ $\mathbf{M}^{\prime}, G: \mathbf{M}^{\prime} \longrightarrow \mathbf{M}$, the objects $U \in \mathbf{M}, U^{\prime} \in \mathbf{M}^{\prime}$ and the induced functors $F / U$ : $\mathbf{M} / U \longrightarrow \mathbf{M}^{\prime} / U^{\prime}, G / U^{\prime}: \mathbf{M}^{\prime} / U^{\prime} \longrightarrow \mathbf{M} / U$ be as in Lemma 4.2. Suppose that $(F, G)$ 
is a Quillen pair for the model categories $\mathbf{M}$ and $\mathbf{M}^{\prime}$. Then, $\left(F / U, G^{\prime} / U^{\prime}\right)$ is a Quillen pair for $\mathbf{M} / U$ and $\mathbf{M}^{\prime} / U^{\prime}$.

Proof. From Example (4) in Section 3, it follows that $\mathbf{M} / U$ and $\mathbf{M}^{\prime} / U^{\prime}$ are fibred categories over $\mathbf{M}$ and $\mathbf{M}^{\prime}$ respectively, and they carry model structures lifted from $\mathbf{M}$ and $\mathbf{M}^{\prime}$. From Lemma 4.2, it follows that $F / U: \mathbf{M} / U \longrightarrow \mathbf{M}^{\prime} / U^{\prime}$ and $G / U^{\prime}: \mathbf{M}^{\prime} / U^{\prime} \longrightarrow$ $\mathbf{M} / U$ are adjoint functors. Then, Proposition 4.1 shows that $F / U$ and $G / U^{\prime}$ are a Quillen pair.

We will now consider fibred model categories with monoidal structures.

Definition 4.4. Suppose that $\underline{\mathbf{N}}=(p: \mathbf{N} \longrightarrow \mathbf{M}), \underline{\mathbf{N}}^{\prime}=\left(p^{\prime}: \mathbf{N}^{\prime} \longrightarrow \mathbf{M}^{\prime}\right)$ and $\underline{\mathbf{N}}^{\prime \prime}=$ $\left(p^{\prime \prime}: \mathbf{N}^{\prime \prime} \longrightarrow \mathbf{M}^{\prime \prime}\right)$ are fibred categories. Then, an adjunction of two variables from $\underline{\mathbf{N}} \times \underline{\mathbf{N}}^{\prime}$ to $\underline{\mathbf{N}}^{\prime \prime}$ is a tuple $\left(\underline{\otimes}, \underline{\operatorname{Hom}}_{l}, \underline{\operatorname{Hom}}_{r}, \underline{\varphi}_{l}, \underline{\varphi}_{r}\right)$ such that $\underline{\otimes}=(\tilde{\otimes}, \otimes), \underline{\operatorname{Hom}}_{l}=$ $\left(\widetilde{\operatorname{Hom}_{l}}, \operatorname{Hom}_{l}\right), \underline{\operatorname{Hom}}_{r}=\left(\widetilde{\operatorname{Hom}_{r}}, \operatorname{Hom}_{r}\right), \underline{\varphi}_{l}=\left(\tilde{\varphi}_{l}, \varphi_{l}\right), \underline{\varphi}_{r}=\left(\tilde{\varphi}_{r}, \varphi_{r}\right)$ where:

(1) $\tilde{\otimes}: \mathbf{N} \times \mathbf{N}^{\prime} \longrightarrow \mathbf{N}^{\prime \prime}, \widetilde{\operatorname{Hom}_{l}}: \mathbf{N}^{o p} \times \mathbf{N}^{\prime \prime} \longrightarrow \mathbf{N}^{\prime}$ and $\widetilde{\operatorname{Hom}_{r}}: \mathbf{N}^{\prime o p} \times \mathbf{N}^{\prime \prime} \longrightarrow \mathbf{N}$ are bifunctors, and $\tilde{\varphi}_{l}$ and $\tilde{\varphi}_{r}$ are natural isomorphisms such that we have an adjunction of two variables from $\mathbf{N} \times \mathbf{N}^{\prime}$ to $\mathbf{N}^{\prime \prime}$ :

$$
\mathbf{N}\left(\xi, \widetilde{\operatorname{Hom}}_{r}\left(\xi^{\prime}, \xi^{\prime \prime}\right)\right) \underset{\tilde{\varphi}_{r}^{-1}}{\cong} \mathbf{N}^{\prime \prime}\left(\xi \tilde{\otimes} \xi^{\prime}, \xi^{\prime \prime}\right) \stackrel{\tilde{\varphi}_{l}}{\cong} \mathbf{N}^{\prime}\left(\xi^{\prime}, \widetilde{\operatorname{Hom}}_{l}\left(\xi, \xi^{\prime \prime}\right)\right)
$$

for objects $\xi \in \mathbf{N}, \xi^{\prime} \in \mathbf{N}^{\prime}$ and $\xi^{\prime \prime} \in \mathbf{N}^{\prime \prime}$.

(2) The bifunctors $\tilde{\otimes}, \widetilde{\mathrm{Hom}}_{l}$ and $\widetilde{\mathrm{Hom}}_{r}$ descend to the bifunctors $\otimes: \mathbf{M} \times \mathbf{M}^{\prime} \longrightarrow$ $\mathbf{M}^{\prime \prime}, \operatorname{Hom}_{l}: \mathbf{M}^{o p} \times \mathbf{M}^{\prime \prime} \longrightarrow \mathbf{M}^{\prime}$ and $\mathrm{Hom}_{r}: \mathbf{M}^{\prime o p} \times \mathbf{M}^{\prime \prime} \longrightarrow \mathbf{M}$ resp. on the base categories and so do the isomorphisms $\tilde{\varphi}_{l}$ and $\tilde{\varphi}_{l}$ to natural isomorphisms $\varphi_{l}$ and $\varphi_{r}$, which form an adjunction of two variables from $\mathbf{M} \times \mathbf{M}^{\prime}$ to $\mathbf{M}^{\prime \prime}$ :

$$
\mathbf{M}\left(U, \operatorname{Hom}_{r}\left(U^{\prime}, U^{\prime \prime}\right)\right) \stackrel{\varphi_{r}^{-1}}{\cong} \mathbf{M}^{\prime \prime}\left(U \otimes U^{\prime}, U^{\prime \prime}\right) \stackrel{\varphi_{l}}{\cong} \mathbf{M}^{\prime}\left(U^{\prime}, \operatorname{Hom}_{l}\left(U, U^{\prime \prime}\right)\right)
$$

for objects $U \in \mathbf{M}, U^{\prime} \in \mathbf{M}^{\prime}$ and $U^{\prime \prime} \in \mathbf{M}^{\prime \prime}$.

We will say that a monoidal structure on a fibred category $\underline{\mathbf{N}}=(p: \mathbf{N} \longrightarrow \mathbf{M})$ consists of a tuple $(\underline{\otimes}, \underline{a}, \underline{l}, \underline{r})$ with $\underline{\otimes}=(\tilde{\otimes}, \otimes), \underline{a}=(\tilde{a}, a), \underline{l}=(\tilde{l}, l)$ and $\underline{r}=(\tilde{r}, r)$ such that

(a) $\tilde{\otimes}: \mathbf{N} \times \mathbf{N} \longrightarrow \mathbf{N}$ is a bifunctor defining a monoidal structure on $\mathbf{N}$ with the associativity isomorphism $\tilde{a}:\left(\xi \otimes \xi^{\prime}\right) \otimes \xi^{\prime \prime \prime} \cong \xi \otimes\left(\xi^{\prime} \otimes \xi^{\prime \prime}\right)$, a unit object $S_{N} \in$ $\mathbf{N}$, a left unit homomorphim $\tilde{l}: S_{N} \otimes \xi \stackrel{\cong}{\longrightarrow} \xi$ and a right unit homomorphism $\tilde{r}: \xi \otimes S_{N} \stackrel{\cong}{\longrightarrow} \xi$ for objects $\xi, \xi^{\prime}$ and $\xi^{\prime \prime} \in \mathbf{N}$.

(b) $\otimes: \mathbf{M} \times \mathbf{M} \longrightarrow \mathbf{M}$ is a bifunctor defining a monoidal structure on $\mathbf{M}$ with the associativity isomorphism $a:\left(U \otimes U^{\prime}\right) \otimes U^{\prime \prime \prime} \stackrel{\cong}{\longrightarrow} U \otimes\left(U^{\prime} \otimes U^{\prime \prime}\right)$, a unit object $S_{M} \in \mathrm{M}$, a left unit homomorphism $l: S_{M} \otimes U \stackrel{\cong}{\longrightarrow} U$ and a right unit homomorphism $r: U \otimes S_{M} \cong U$ for objects $U, U^{\prime}$ and $U^{\prime \prime} \in \mathbf{M}$.

(c) The bifunctor $\tilde{\otimes}: \mathbf{N} \times \mathbf{N} \longrightarrow \mathbf{N}$ descends to the bifunctor $\otimes: \mathbf{M} \times \mathbf{M} \longrightarrow \mathbf{M}$ on the base category; the isomorphisms $\tilde{a}, \tilde{l}$ and $\tilde{r}$ descend to the isomorphisms $a, l$ and $r$ respectively, and $p\left(S_{N}\right)=S_{M}$. 
We can now define what a closed monoidal structure on a fibred category should be. For the usual definition of a closed monoidal structure on an ordinary category, see $[2, \S 4.1 .13]$.

Definition 4.5. A closed monoidal structure on a fibred category $\underline{\mathbf{N}}=(p: \mathbf{N} \longrightarrow \mathbf{M})$ consists of a tuple

$$
\left(\underline{Q}, \underline{a}, \underline{l}, \underline{r}, \underline{\operatorname{Hom}}_{l}, \underline{\operatorname{Hom}}_{r}, \underline{\varphi}_{l}, \underline{\varphi}_{r}\right)
$$

such that $(\underline{\otimes}, \underline{a}, \underline{l}, \underline{r})$ defines a monoidal structure on $\underline{\mathbf{N}}$, and $\left(\underline{\otimes}, \underline{\operatorname{Hom}}_{l}, \underline{\operatorname{Hom}}_{r}, \underline{\varphi}_{l}, \underline{\varphi}_{r}\right)$ defines an adjunction of two variables from $\underline{\mathbf{N}} \times \underline{\mathbf{N}}$ to $\underline{\mathbf{N}}$ in the sense of Definition $\overline{4} .4$.

We recall that an adjunction $\left(\otimes, \operatorname{Hom}_{l}, \operatorname{Hom}_{r}, \varphi_{l}, \varphi_{r}\right)$ of two variables from $\mathbf{M} \times \mathbf{M}$ to $\mathbf{M}$ for a model category $\mathbf{M}$ is called a Quillen adjunction of two variables (see [2, $\S 4.2 .1])$ if, given two cofibrations $f: U \longrightarrow V$ and $g: W \longrightarrow X$ in $\mathbf{M}$, the induced map

$$
f \square g:=P(f, g)=(V \otimes W) \coprod_{U \otimes W}(U \otimes X) \longrightarrow V \otimes X
$$

is a cofibration, which is trivial if one of $f$ and $g$ is. For sake of brevity, we will often abuse notation to refer to the tuple $\left(\otimes, a, l, r, \operatorname{Hom}_{l}, \operatorname{Hom}_{r}, \varphi_{l}, \varphi_{r}\right)$, defining both a Quillen adjunction of two variables and a closed monoidal structure on $\mathbf{M}$ as a Quillen bifunctor $\otimes$ on the model category $\mathbf{M}$.

As usual, for the fibred model category $(\mathbf{N}, p, \mathbf{M})$, we will use $(\mathbf{C}, \mathbf{W}, \mathbf{F})$ to denote the model structure on the base category $\mathbf{M}$ and $\left(\mathbf{C}_{N}, \mathbf{W}_{N}, \mathbf{F}_{N}\right)$ to denote the lifted model structure on $\mathbf{N}$. The following proposition now shows that given a closed monoidal structure $\left(\underline{\otimes}, \underline{a}, \underline{l}, \underline{r}, \underline{\operatorname{Hom}}_{l}, \underline{\operatorname{Hom}}_{r}, \underline{\varphi}_{l}, \underline{\varphi}_{r}\right)$ on a fibred model category $(\mathbf{N}, p, \mathbf{M})$ and a Quillen bifunctor $\otimes$ for a model structure on the base category $\mathbf{M}$, $\tilde{\otimes}$ becomes a Quillen bifunctor on $\mathbf{N}$ if the functor $p$ commutes with colimits.

Proposition 4.6. Suppose that $\left(\underline{\otimes}, \underline{a}, \underline{l}, \underline{r}, \underline{\operatorname{Hom}}_{l}, \underline{\operatorname{Hom}}_{r}, \underline{\varphi}_{l}, \underline{\varphi}_{r}\right)$ is a closed monoidal structure on a fibred model category $(\mathbf{N}, p, \mathbf{M})$ in the notation of Definition 4.5. Furthermore, assume that the functor $p: \mathbf{N} \longrightarrow \mathbf{M}$ commutes with finite colimits. Then, if the tuple $\left(\otimes, a, l, r, \operatorname{Hom}_{l}, H_{o m}, \varphi_{l}, \varphi_{r}\right)$ defines a Quillen bifunctor $\otimes$ on the base category $\mathbf{M}$, the tuple $\left(\tilde{\otimes}, \tilde{a}, \tilde{l}, \tilde{r}, \widehat{\operatorname{Hom}}_{l}, \widehat{\operatorname{Hom}}_{r}, \tilde{\varphi}_{l}, \tilde{\varphi}_{r}\right)$ defines a Quillen bifunctor $\tilde{\otimes}$ for the lifted model structure on $\mathbf{N}$.

Proof. We will maintain the notation of Definition 3.1. Suppose that $\phi: \xi \longrightarrow \xi^{\prime}$ and $\varphi: \eta \longrightarrow \eta^{\prime}$ are two cofibrations in $\mathbf{N}$, i.e., morphisms in $\mathbf{C}_{N}$. Consider the pushout product

$$
\phi \square \varphi: \xi^{\prime} \tilde{\otimes} \eta \coprod_{\xi \tilde{\otimes} \eta} \xi \tilde{\otimes} \eta^{\prime} \longrightarrow \xi^{\prime} \tilde{\otimes} \eta^{\prime}
$$

and its image in $\mathbf{M}$, which is also a pushout product (since $p$ commutes with finite colimits)

$$
p(\phi \square \varphi)=p(\phi) \square p(\varphi): p\left(\xi^{\prime}\right) \otimes p(\eta) \coprod_{p(\xi) \otimes p(\eta)} p(\xi) \otimes p\left(\eta^{\prime}\right) \longrightarrow p\left(\xi^{\prime}\right) \otimes p\left(\eta^{\prime}\right) .
$$

Since $\phi: \xi \longrightarrow \xi^{\prime}$ and $\varphi: \eta \longrightarrow \eta^{\prime}$ lie in $\mathbf{C}_{N}$, it follows that $p(\phi): p(\xi) \longrightarrow p\left(\xi^{\prime}\right)$ and $p(\varphi): p(\eta) \longrightarrow p\left(\eta^{\prime}\right)$ lie in $\mathbf{C}$. Since $\otimes$ is a Quillen bifunctor on $\mathbf{M}$, it follows that 
$p(\phi \square \varphi)=p(\phi) \square p(\varphi)$ is a cofibration in $\mathbf{M}$, whence it follows that $\phi \square \varphi$ lies in $\mathbf{C}_{N}$. Moreover, if any of $\phi$ or $\varphi$ lies in $\mathbf{W}_{N}$, then it follows that its image under $p$ lies in $\mathbf{W}$ and hence $p(\phi \square \varphi)$ lies in both $\mathbf{C}$ and $\mathbf{W}$. It follows that $\phi \square \varphi$ is also a trivial cofibration in $\mathbf{N}$, i.e., it lies in both $\mathbf{C}_{N}$ and $\mathbf{W}_{N}$.

Let $\left(\underline{\otimes}, \underline{a}, \underline{l}, \underline{r}, \underline{\operatorname{Hom}}_{l}, \underline{\operatorname{Hom}}_{r}, \underline{\varphi}_{l}, \underline{\varphi}_{r}\right)$ be a closed monoidal structure on the fibred category $\underline{\mathbf{N}}=(\mathbf{N}, p, \mathbf{M})$ and $\otimes$ be a Quillen bifunctor for a model structure on the base category $\mathbf{M}$. We will assume that $p: \mathbf{N} \longrightarrow \mathbf{M}$ commutes with colimits, and hence Proposition 4.6 shows that $\tilde{\otimes}$ is a Quillen bifunctor for the lifted model structure on $\mathbf{N}$. We will say that the above defines a Quillen bifunctor $\underline{\otimes}: \underline{\mathbf{N}} \times \underline{\mathbf{N}} \longrightarrow \underline{\mathbf{N}}$.

Finally, we recall that a monoidal model category $\mathbf{M}$ consists of a closed monoidal category with a model structure such that

(a) The monoidal structure $\otimes: \mathbf{M} \times \mathbf{M} \longrightarrow \mathbf{M}$ is a Quillen bifunctor.

(b) Given the cofibrant replacement $Q S \stackrel{q}{\longrightarrow} S$ for the unit $S$ of the monoidal structure $\otimes$, the natural morphisms $Q S \otimes X \longrightarrow S \otimes X$ and $X \otimes Q S \longrightarrow X \otimes S$ are weak equivalences for all cofibrant objects $X$ in $\mathbf{M}$.

Therefore, we define a monoidal fibred model category as follows:

Definition 4.7. Let $\underline{\mathbf{N}}=(\mathbf{N}, p, \mathbf{M})$ be a fibred model category with a closed monoidal structure given by a tuple $\left(\underline{\otimes}, \underline{a}, \underline{l}, \underline{r}, \underline{\operatorname{Hom}}_{l}, \underline{\operatorname{Hom}}_{r}, \underline{\varphi}_{l}, \underline{\varphi}_{r}\right)$ in the sense of Definition 4.5. Then, we will say that $\underline{\mathbf{N}}$ is a monoidal fibred model category if:

(a) The monoidal structure $\underline{\otimes}: \underline{\mathbf{N}} \times \underline{\mathbf{N}} \longrightarrow \underline{\mathbf{N}}$ is a Quillen bifunctor.

(b) Given the cofibrant replacement $Q_{N} S_{N} \stackrel{q_{N}}{\longrightarrow} S_{N}$ (resp. $Q_{M} S_{M} \stackrel{q_{M}}{\longrightarrow} S_{M}$ ) for the unit $S_{N}$ (resp. $\left.S_{M}\right)$ of the monoidal structure $\tilde{\otimes}($ resp. $\otimes)$ on $\mathbf{N}$ (resp. $\mathbf{M}$ ), the natural morphism $Q_{N} S_{N} \tilde{\otimes} \xi \longrightarrow S_{N} \tilde{\otimes} \xi$ (resp. $Q_{M} S_{M} \otimes U \longrightarrow S_{M} \otimes U$ ) is a weak equivalence for all cofibrant objects $\xi$ (resp. $U$ ) in $\mathbf{N}$ (resp. M). Similarly, the natural morphism $\xi \tilde{\otimes} Q_{N} S_{N} \longrightarrow \xi \tilde{\otimes} S_{N}$ (resp. $U \otimes Q_{M} S_{M} \longrightarrow U \otimes S_{M}$ ) is a weak equivalence for all cofibrant objects $\xi$ (resp. $U$ ) in $\mathbf{N}$ (resp. $\mathbf{M}$ ).

The last proposition now shows that a monoidal model structure on the base category $\mathbf{M}$ also lifts to the category $\mathbf{N}$, giving $\underline{\mathbf{N}}$ the structure of a monoidal fibred model category.

Proposition 4.8. Suppose that $\left(\underline{\otimes}, \underline{a}, \underline{l}, \underline{r}, \underline{\operatorname{Hom}}_{l}, \underline{\operatorname{Hom}}_{r}, \underline{\varphi}_{l}, \underline{\varphi}_{r}\right)$ is a closed monoidal structure on a fibred model category $(\mathbf{N}, p, \mathbf{M})$ in the notation of Definition 4.5. Furthermore, assume that the functor $p: \mathbf{N} \longrightarrow \mathbf{M}$ commutes with finite colimits. Then, if the tuple $\left(\otimes, a, l, r, \operatorname{Hom}_{l}, \operatorname{Hom}_{r}, \varphi_{l}, \varphi_{r}\right)$ makes the base category $\mathbf{M}$ into a monoidal model category, the tuple $\left(\underline{\otimes}, \underline{a}, \underline{l}, \underline{r}, \underline{\operatorname{Hom}}_{l}, \underline{\operatorname{Hom}}_{r}, \underline{\varphi}_{l}, \underline{\varphi}_{r}\right)$ makes $\underline{\mathbf{N}}=(\mathbf{N}, p, \mathbf{M})$ into a monoidal fibred model category.

Proof. From Proposition 4.6, it follows already that $\underline{\mathbb{Q}}: \underline{\mathbf{N}} \times \underline{\mathbf{N}} \longrightarrow \underline{\mathbf{N}}$ is a Quillen bifunctor.

Suppose that $\xi$ is a cofibrant object of $\mathbf{N}$, i.e., $0 \longrightarrow \xi$ is a cofibration in $\mathbf{N}$. Hence, $0 \longrightarrow p(\xi)$ is a cofibration in $\mathbf{M}$. It follows that $Q_{M} S_{M} \otimes p(\xi) \longrightarrow S_{M} \otimes p(\xi)$ is a weak equivalence in $\mathbf{M}$. From Corollary 3.6, it follows that $Q_{M} S_{M}=p\left(Q_{N} S_{N}\right)$, and hence $p\left(Q_{N} S_{N} \tilde{\otimes} \xi \longrightarrow S_{N} \tilde{\otimes} \xi\right)=Q_{M} S_{M} \otimes p(\xi) \longrightarrow S_{M} \otimes p(\xi)$. It follows that $Q_{N} S_{N} \tilde{\otimes} \xi \longrightarrow S_{N} \tilde{\otimes} \xi$ is a weak equivalence in N. Similarly, $\xi \tilde{\otimes} Q_{N} S_{N} \longrightarrow \xi \tilde{\otimes} S_{N}$ is a weak equivalence in $\mathbf{N}$. This proves the result. 


\section{References}

[1] D.G. Quillen, Homotopical algebra, Lecture Notes in Mathematics 43, SpringerVerlag, New York, 1967.

[2] M. Hovey, Model categories, Mathematical Surveys and Monographs, No. 63, Amer. Math. Soc., Providence, RI, 1999.

[3] M. Hovey, Cotorsion pairs and model categories, in Interactions between homotopy theory and algebra, Contemp. Math. 436, Amer. Math. Soc., Providence, RI, 2007, 277-296.

[4] J. Gillespie, The flat model structure on complexes of sheaves, Trans. Amer. Math. Soc. 358 (2006), no. 7, 2855-2874.

[5] J.F. Jardine, Simplicial presheaves, J. Pure Appl. Algebra 47 (1987), no. 1, 3587.

[6] A. Grothendieck et al., Revêtements étales et groupe fondamental, Séminaire de Géométrie Algébrique du Bois Marie 1960-1961 (SGA 1), Dirigé par Alexandre Grothendieck. Augmenté de deux exposés de M. Raynaud. Lecture Notes in Mathematics 224, Springer-Verlag, New York, 1971.

[7] A. Canonaco, Introduction to algebraic stacks, preprint (2004).

[8] A. Vistoli, Notes on Grothendieck topologies, fibered categories and descent theory, in (B. Fantechi, L. Gottsche, L. Illusie, S. Kleiman, N. Nitsure, A. Vistoli, eds.), Fundamental algebraic geometry: Grothendieck's FGA explained, Mathematical Surveys and Monographs 123, Amer. Math. Soc., Providence, RI, 2006.

Abhishek Banerjee abhishekbanerjee1313@gmail.com

Department of Mathematics, The Ohio State University, 231 West 18th Avenue, Columbus, OH 43210-1174, USA 